\title{
Flare-out conditions in static thin-shell wormholes
}

\author{
S. Habib Mazharimousavi ${ }^{*}$ and M. Halilsoy ${ }^{\dagger}$ \\ Department of Physics, Eastern Mediterranean University, \\ Gazimă̆usa, via Mersin 10, north Cyprus, Turkey \\ (Received 12 June 2014; published 10 October 2014)
}

\begin{abstract}
We reconsider the generalized flare-out conditions in static wormhole throats given by Hochberg and Visser. We show that, due to the presence of matter sources on the throat, these conditions are not applicable to the thin-shell wormholes.
\end{abstract}

DOI: $10.1103 /$ PhysRevD.90.087501

PACS numbers: $04.20 . \mathrm{Gz}, 04.20 . \mathrm{Cv}$

\section{INTRODUCTION}

Much has been written about traversable wormholesthe shortcut hypothetical channels-between distant points of the same or different universes. A safe passage is assumed provided the tunnel resists collapse for a considerable time. Repulsion against gravitational collapse is provided by a negative energy density that is absent in large amounts in our world. Although at atomic scales quantum theory comes to our rescue such scales are for elementary particles/photons, not for humans. Let us add that modified Einstein theories admit wormholes that are supported by normal, nonexotic matter. The difficulty is finding similar objects in the simplest theory of gravity, namely Einstein's general relativity. Exploring such structures relies on our understanding of the physics of the tunnel and its narrowest surface: the throat. It was suggested first by Morris and Thorne [1] that the minimal two-dimensional surface of the throat must satisfy the flare-out conditions. This ensured connection to distant points provided the points belong to asymptotically flat universes. This is an ideal case that can be relaxed, i.e., even for nonasymptotically flat spaces construction of wormholes may be taken for granted.

The flare-out conditions for a throat set by Morris and Thorne [1] were generalized by Hochberg and Visser [2]. In the latter, known as the generalized Morris-Thorne flare-out conditions, they employ the extrinsic curvature tensor $K_{a b}$ and its trace, $\operatorname{tr}(K)=g^{a b} K_{a b}$, for $(a, b) \in \Sigma$, the twodimensional geometry of the throat. In brief it states that the area of the throat $A(\Sigma)$ satisfies both $\delta A=0$ and $\delta^{2} A \geq 0$ for the minimality requirement. These amount to $\operatorname{tr}(K)=0$ and $\frac{\partial(\operatorname{tr}(K))}{\partial n} \leq 0$, for the normal direction $n$ to the twodimensional geometry of the throat.

We show in this article that the generalized MorrisThorne flare-out conditions introduced for a wormhole are not applicable to a thin-shell wormhole (TSW), which is constructed by the cut-and-paste technique. For this purpose we split the geometry in Gaussian normal coordinates into $T \times \Sigma$ and apply the Israel junction conditions [3]. The very existence of surface energy density $\sigma$ at the junction of

\footnotetext{
*habib.mazhari@emu.edu.tr

†mustafa.halilsoy@emu.edu.tr
}

the TSW violates $\operatorname{tr}(K)=0$ condition but yet the minimality of the area can be preserved. This is not as a result of a mathematical proof but rather as a requirement to define the existence of a throat. The distinction can be seen in Fig. 1 between a normal wormhole and a TSW. In the latter case the lack of smoothness at the throat prevents the metric functions to admit continuous derivatives. For the TSW we proceed to propose the condition $\operatorname{tr}(K) \gtrless 0$, irrespective of the minimality of area. This yields further that the surface energy-density $\sigma$ can locally be negative/positive, but more importantly its total (i.e., the integral of $\sigma$ ), which matters physically may turn out to be positive. This is shown by application to the nonspherical Zipoy-Voorhees (ZV) $[4,5]$ metric through numerical plots for tuned parameters.

\section{WEAKER FLARE-OUT CONDITIONS FOR TSWS}

Hochberg and Visser, [2], generalized the minimal area condition for wormholes given previously by Morris and Thorne in [1]. Based on [2] an arbitrary static spacetime (which is supposed to be a wormhole) can be written as

$$
d s^{2}=g_{\mu \nu} d x^{\mu} d x^{\nu}=-\exp (2 \phi) d t^{2}+g_{i j}^{(3)} d x^{i} d x^{j}
$$

in which $\mu, \nu=0,1,2,3$ while $i, j=1,2,3$ and $\phi=\phi\left(x^{i}\right)$. The definition of a throat for the traversable wormhole, following [1,2], is given to be a two-dimensional
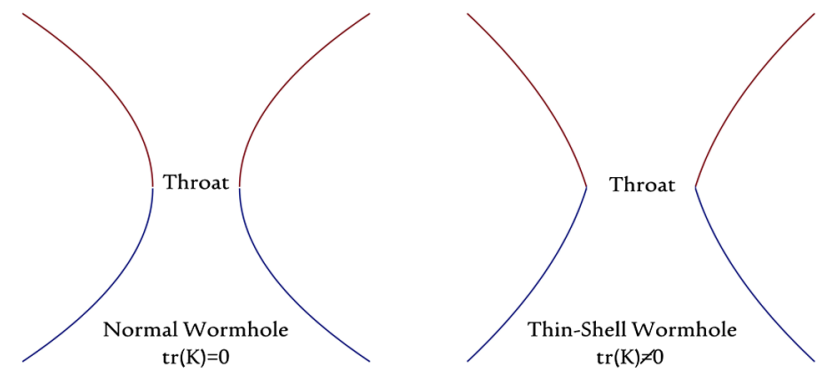

FIG. 1 (color online). A plot of a normal wormhole and thinshell wormhole. The existence of matter at the throat of a thinshell wormhole causes $\operatorname{tr}(K) \neq 0$ unlike in the normal wormhole. We also add that the original minimum area condition of Morris Thorne for both cases is applicable. 
hypersurface $\Sigma$ of minimal area taken in one of the constant-time spatial slices. The area of the throat is given by

$$
A(\Sigma)=\int \sqrt{g^{(2)}} d^{2} x .
$$

A further step is taken if one uses the Gaussian normal coordinates with $x^{i}=\left(x^{a}, n\right)$ and rewrites

$$
g_{i j}^{(3)} d x^{i} d x^{j}=g_{a b}^{(2)} d x^{a} d x^{b}+d n^{2}
$$

Now, the question is "are the generalized Morris-Thorne flare-out conditions [2] applicable to a TSW?" To find the answer we start from the beginning. Let us consider an arbitrary static four-dimensional spacetime of the form

$$
d s^{2}=g_{\mu \nu}^{(4)} d x^{\mu} d x^{\nu}=g_{00} d t^{2}+g_{i j}^{(3)} d x^{i} d x^{j}
$$

We note that here in (4) $g_{00}$ may have root(s) or not and, if yes, we call $r=r_{h}$ the largest root, or event horizon. Then we use the standard method of making TSW [6]. The throat is located at the hypersurface $x^{i}=a$ where $i$ can be only one of the possible spatial coordinates. Without loss of generality we set $i=1$ and the line element of the throat reads as

$$
d s_{\Sigma}^{2}=-d \tau^{2}+g_{a b}^{(2)} d x^{a} d x^{b}
$$

in which $a, b=2,3$. The full line element of the throat in a TSW, by definition, is in the form of the Gaussian normal coordinates [7] and therefore the area of the throat is given by (2). Having minimum spatial area for the throat, hence, requires the same procedure as introduced in [2], i.e., $\delta A(\Sigma)=0$ and $\delta^{2} A(\Sigma) \geq 0$ which ultimately end up with the same conditions as the ordinary wormholes, i.e., $\operatorname{tr}(K)=0$ and $\frac{\partial \operatorname{tr}(K)}{\partial n} \leq 0$. Further study on TSWs, however, manifests that unlike the case of an ordinary wormhole spacetime, in TSW we are allowed to have some matter sources on the throat. The matter supporting the throat should satisfy the standard Israel junction conditions [3] or Einstein equations on the throat,

$$
\left\langle K_{i}^{j}\right\rangle-\langle K\rangle \delta_{i}^{j}=-8 \pi S_{i}^{j}
$$

in which $\langle$.$\rangle stands for a jump across the hypersurface \Sigma$, $\langle K\rangle=\left\langle K_{i}^{i}\right\rangle$, and $S_{i}^{j}=\operatorname{diag}\left(-\sigma, p_{2}, p_{3}\right)$ with $i, j=\tau, 2,3$. $\operatorname{The} \operatorname{tr}(K)$ that must be zero is just the trace of spatial part of the extrinsic curvature tensor (6) that corresponds to the space part of Eq. (5),

$$
K_{a}^{b}=\left(\begin{array}{cc}
\left\langle K_{2}^{2}\right\rangle & 0 \\
0 & \left\langle K_{3}^{3}\right\rangle
\end{array}\right),
$$

i.e., $\operatorname{tr}(K)=\left\langle K_{2}^{2}\right\rangle+\left\langle K_{3}^{3}\right\rangle$. We note that, as it was used in [2], $\operatorname{tr}(K)$ refers to the trace of extrinsic curvature of the spatial part of Gaussian line element (5), i.e., $\operatorname{tr}(K)=\left\langle K_{a}^{a}\right\rangle$, while $\langle K\rangle$ implies the trace of the $2+1$-dimensional Gaussian line element of the thin-shell wormhole, i.e., $\langle K\rangle=\left\langle K_{i}^{i}\right\rangle$. In other words, $\operatorname{tr}(K)+\left\langle K_{\tau}^{\tau}\right\rangle=\langle K\rangle$.

Looking closely at (6), one finds the $\tau \tau$ component to be

$$
\left\langle K_{\tau}^{\tau}\right\rangle-\langle K\rangle=8 \pi \sigma
$$

or after considering the $2+1$-dimensional trace implied in (6) $\langle K\rangle=\left\langle K_{\tau}^{\tau}\right\rangle+\left\langle K_{2}^{2}\right\rangle+\left\langle K_{3}^{3}\right\rangle$ this becomes

$$
\left\langle K_{2}^{2}\right\rangle+\left\langle K_{3}^{3}\right\rangle=-8 \pi \sigma
$$

The left-hand side is nothing but $\operatorname{tr}(K)$, which is supposed to vanish at the throat. In general $\sigma \neq 0$, which violates $\operatorname{tr}(K)=0$ and simply means that the generalized MorrisThorne flare-out conditions are not applicable to the TSW. However, the latter condition does not change the applicability of the original Morris-Thorne's minimality conditions. In Fig. 1 we see the implication of $\operatorname{tr}(K) \neq 0$ for a TSW, since derivatives of the metric function are not continuous at the throat. Let us add that $\operatorname{tr}(K)=0$ also indirectly stands for the ordinary wormholes whose throat surfaces trivially have no external energy momenta.

Based on what we found, for the TSWs in general $\operatorname{tr}(K) \neq 0$ while the area of the throat can still be minimum. Nevertheless, $\operatorname{tr}(K)>0 / \operatorname{tr}(K)<0$ strongly suggests that $\sigma<0 / \sigma>0$ on the throat.

\section{ILLUSTRATIVE EXAMPLES}

Next, we consider some explicit examples studied in the literature. The first example is the TSW in Schwarzschild spacetime given by Poisson and Visser [7]. In that case

$$
g_{a b}^{(2)}=\left(\begin{array}{cc}
a^{2} & 0 \\
0 & a^{2} \sin (\theta)
\end{array}\right),
$$

and the throat is located at $r=a$. The extrinsic curvature of 2-surface is found to be

$$
K_{a}^{b}=\left(\begin{array}{cc}
\frac{2}{a} \sqrt{1-\frac{2 M}{a}} & 0 \\
0 & \frac{2}{a} \sqrt{1-\frac{2 M}{a}}
\end{array}\right)
$$

with $\operatorname{tr}(K)=\frac{4}{a} \sqrt{1-\frac{2 M}{a}}$ at the throat, which is clearly positive. This can be seen when we recall that $a>r_{h}=2 M$.

For the second example we consider the cylindrically symmetric TSW studied in [8]. The bulk metric is given by

$$
d s^{2}=f(r)\left(-d t^{2}+d r^{2}\right)+h(r) d z^{2}+g(r) d \varphi^{2}
$$


and the throat is located at $r=a$ with the line element

$$
d s_{\Sigma}^{2}=-d \tau^{2}+h(a) d z^{2}+g(a) d \varphi^{2}
$$

therefore, we have

$$
g_{a b}^{(2)}=\left(\begin{array}{cc}
h(a) & 0 \\
0 & g(a)
\end{array}\right) .
$$

As it was found in [8] one finds

$$
K_{a}^{b}=\left(\begin{array}{cc}
\frac{h^{\prime}}{h \sqrt{f}} & 0 \\
0 & \frac{g^{\prime}}{g \sqrt{f}}
\end{array}\right)
$$

in which a prime stands for the derivative with respect to $r$ and all functions are found at $r=a$. The trace of the extrinsic curvature is given by

$$
\operatorname{tr}(K)=\frac{h^{\prime}}{h \sqrt{f}}+\frac{g^{\prime}}{g \sqrt{f}},
$$

which in general is not zero. For instance, one of the cases in [8] is the straight cosmic string with $f=1, g=W_{0} r^{2}$ and $h=1$ with $\operatorname{tr}(K)=\frac{2}{a}$, which is not zero but positive.

Our last example has been introduced in [5], which is the TSW in ZV spacetime [4]. The bulk metric of ZV is given by

$d s^{2}=-A(x) d t^{2}+B(x, y) d x^{2}+C(x, y) d y^{2}+F(x, y) d \varphi^{2}$,

where

$$
\begin{aligned}
A(x) & =\left(\frac{x-1}{x+1}\right)^{\delta} \\
B(x, y) & =k^{2}\left(\frac{x+1}{x-1}\right)^{\delta}\left(\frac{x^{2}-1}{x^{2}-y^{2}}\right)^{\delta^{2}}\left(\frac{x^{2}-y^{2}}{x^{2}-1}\right) \\
C(x, y) & =k^{2}\left(\frac{x+1}{x-1}\right)^{\delta}\left(\frac{x^{2}-1}{x^{2}-y^{2}}\right)^{\delta^{2}}\left(\frac{x^{2}-y^{2}}{1-y^{2}}\right)
\end{aligned}
$$

and

$$
F(x, y)=k^{2}\left(\frac{x+1}{x-1}\right)^{\delta}\left(x^{2}-1\right)\left(1-y^{2}\right),
$$

in which $k=\frac{M}{\delta}$ with $M=$ mass and $\delta$ is the parameter of oblateness. The range of coordinates is $1<x<\infty$, $-1 \leq y \leq 1,0 \leq \varphi \leq 2 \pi$. The throat is located at $x=a=$ const $>1$ and therefore

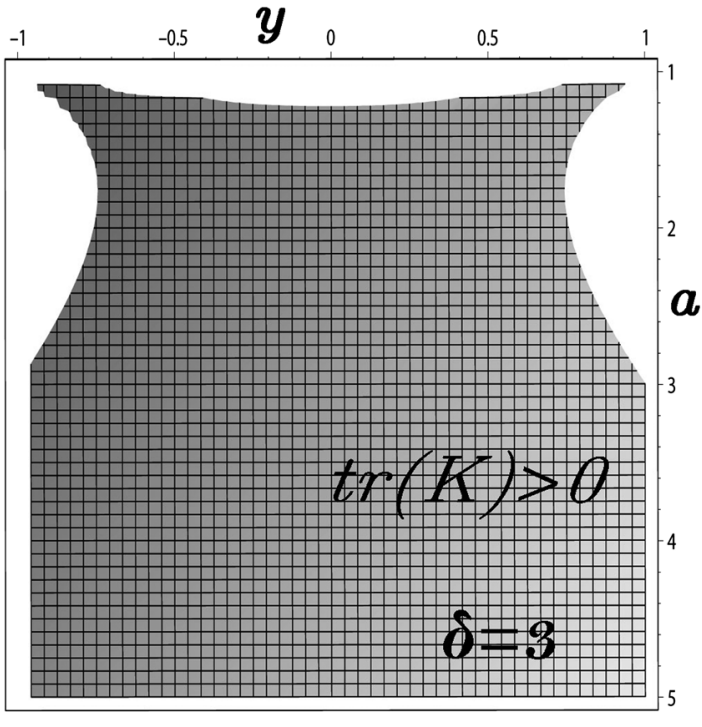

FIG. 2. Extrinsic curvature $\operatorname{tr}(K)$ with respect to $a$ and $y$ for $\delta=3$. As it is seen from the figure for large enough $a, \operatorname{tr}(K)$ is positive on the entire y-axis. For small $a, \operatorname{tr}(K)$ is not positive everywhere on the $y$-axis but it is also not entirely negative on the y-axis.

$$
g_{a b}^{(2)}=\left(\begin{array}{cc}
C(a, y) & 0 \\
0 & F(a, y)
\end{array}\right) .
$$

The extrinsic curvature then reads

$$
K_{a}^{b}=\left(\begin{array}{cc}
\frac{C_{a}}{C \sqrt{B}} & 0 \\
0 & \frac{F_{a}}{F \sqrt{B}}
\end{array}\right)
$$

in which a sub $a$ implies partial derivative with respect to $a$. The trace of (23) is given by

$$
\operatorname{tr}(K)=\frac{C_{a}}{C \sqrt{B}}+\frac{F_{a}}{F \sqrt{B}}=\frac{1}{\sqrt{B}} \frac{\partial}{\partial a} \ln (F C) .
$$

This is a function of $a$ and $y$ that obviously is not zero. In Fig. 2 we plot $\operatorname{tr}(K)$ in terms of $a$ and $y$ for some value of $\delta>2$ that is of interest in [5]. We observe that for large enough $a$ for the entire interval of $y$ the trace of $\operatorname{tr}(K)$ is positive.

\section{CONCLUSION}

The generalized Morris-Thorne flare-out conditions, i.e., $\delta A(\Sigma)=0$ and $\delta^{2} A(\Sigma) \geq 0$ proposed for general wormholes are weakened for the case of TSWs. This is necessary due to the fact that on the TSW at the throat we have a surface energy density $\sigma \neq 0$. Accordingly, this modifies the vanishing of $\operatorname{tr}(K)$. We propose instead that $\operatorname{tr}(K)>0 / \operatorname{tr}(K)<0$, which relates to the sign of the local energy density. Therefore the original minimality of the throat area by Morris and Thorne stays intact by 
construction while the extrinsic curvature tensor has a nonzero trace at the throat. Derivatives of the extrinsic curvature are not continuous at the throat so that the mathematical proof of Ref. [2] cannot be used. The throat can be chosen anywhere through the cut-and paste method beyond singularities or event horizons (if any). This is the strategy that has been adopted in the TSW example of nonspherical ZV spacetime [5].

\section{ACKNOWLEDGMENTS}

The authors thank the anonymous referee for helpful and constructive comments.
[1] M. S. Morris and K. S. Thorne, Am. J. Phys. 56, 395 (1988).

[2] D. Hochberg and M. Visser, Phys. Rev. D 56, 4745 (1997).

[3] W. Israel, Nuovo Cimento B 44, 1 (1966); V. de la Cruz and W. Israel, Nuovo Cimento A 51, 744 (1967); J. E. Chase, Nuovo Cimento B 67, 136 (1970); S. K. Blau, E. I. Guendelman, and A. H. Guth, Phys. Rev. D 35, 1747 (1987); R. Balbinot and E. Poisson, Phys. Rev. D 41, 395 (1990).

[4] H. Weyl, Ann. Phys. (Berlin) 359, 117 (1917); D. M. Zipoy, J. Math. Phys. (N.Y.) 7, 1137 (1966); B. H. Voorhees, Phys. Rev. D 2, 2119 (1970).
[5] S. H. Mazharimousavi and M. Halilsoy, Eur. Phys. J. C 74, 3067 (2014).

[6] M. Visser, Phys. Rev. D 39, 3182 (1989); M. Visser, Nucl. Phys. B328, 203 (1989); P. R. Brady, J. Louko, and E. Poisson, Phys. Rev. D 44, 1891 (1991).

[7] E. Poisson and M. Visser, Phys. Rev. D 52, 7318 (1995); M. Visser, Lorentzian Wormholes from Einstein to Hawking (American Institute of Physics, New York, 1995).

[8] E. F. Eiroa and C. Simeone, Phys. Rev. D 70, 044008 (2004). 